\title{
Les dysrégulations gonadotropes féminines: une réactualisation
}

\author{
Pierre Mauvais-Jarvis
}

Les critères d'action des médicaments largement utilisés en gynécologie, notamment des estroprogestatifs, ne sont-ils pas aujourd'hui dépassés, faisant trop appel à la notion empirique d'efficacité et pas assez à une analyse raisonnée des modes d'action et des éventuels effets secondaires? Telle est la question en forme d'accusation que pose le professeur Pierre Mauvais-Jarvis.

\section{TIRÉS A PART}

P. Mauvais-Jarvis. $\mathrm{m} / \mathrm{s} n^{\circ} 10$, vol. 6 , décembre 90

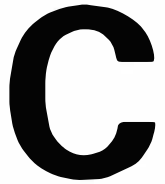
hez la femme normale, un cycle ovulatoire nécessite, pour sa réalisation, une parfaite coordination entre la commande hypothalamique de l'ovulation (générateur hypothalamique) et les rétroactions hormonales ou neurohormonales qui s'exercent au niveau de l'hypothalamus et de l'hypophyse, provenant soit des neurones centraux soit de l'ovaire luimême $[6,12]$. On s'accorde désormais pour reconnaître un rôle primordial à l'estradiol dans le déterminisme de l'ovulation proprement dite ; on sait aussi que l'initiation du cycle menstruel et son déroulement normal résultent de l'activation des neurones hypothalamiques contrôlant la libération pulsatile d'un décapeptide, la GnRH (gonadotropin-releasing hormone) [12]. Cette pulsatilité (une décharge de $\mathrm{GnRH}$ toutes les heures environ) est modulable quant à son amplitude et à sa fréquence, non seulement sous l'effet des stéroïdes ovariens (estradiol et progestérone) [12], mais encore par l'intermédiaire de neuromodulateurs hypothalamiques : dopamine, noradrénaline et surtout opioïdes [6-8]. La destruction, chez le singe rhésus femelle, du noyau arqué de l'hypothalamus médian abolit toute sécrétion de $\mathrm{GnRH}$, et donc de LH (luteinizing hormone) et de FSH (follicle stimulating hormone), par l'hypophyse [12]. Il en résulte une suppression de toute activité hormonale ovarienne, ce que l'on observe d'ailleurs chez l'être humain au cours des agénésies hypothalamiques type Kallman de Morsier [4]. Inversement, l'administration continue de $\mathrm{GnRH}$ synthétique exogène ou d'un agoniste de la GnRH abolit, par désensibilisation des récepteurs hypophysaires de la $\mathrm{GnRH}$, toute sécrétion de $\mathrm{LH}$ et, à un moindre degré, de FSH, permettant de réaliser en thérapeutique une castration biochimique [12].

Entre ces deux situations, des perturbations dans la fréquence ou l'amplitude des pulsations de $\mathrm{GnRH}$ peuvent être génératrices d'une sécrétion inadéquate de FSH et de $\mathrm{LH}$ responsable, à son tour, d'une mauvaise maturation folliculaire, puis d'une décharge ovulatoire insuffisante et, secondairement, d'une insuffisance lutéale [19]. Le syndrome des ovaires polykystiques constitue l'illustration extrême de cette situation pathologique. Schématiquement, dans cette affection, l'amplitude et la fréquence de la sécrétion de $\mathrm{GnRH}$ seraient augmentées. Il en résulterait une élévation du rapport $\mathrm{LH} / \mathrm{FSH}$ sécrétés par l'hypophyse avec augmentation de la sécrétion pulsatile de $\mathrm{LH}$ et diminution de FSH $[15,18]$. Toutefois, bien d'autres anomalies de la pulsatilité de la GnRH peuvent s'observer, dès lors que l'on analyse finement et de façon répétée la sécrétion de LH bioactive et de FSH [13]. Ces altérations créent des situations cliniques qui vont de tableaux relativement proches de l'ovaire polykystique à de simples altérations du cycle menstruel que nous avons 
dénommées, il y a plusieurs années, "dysovulation" [16]. Les données que nous venons d'évoquer nous font facilement entrevoir quelles peuvent être les causes des anomalies de la pulsatilité de GnRH. Si - par exemple - l'hyperproduction d'opioïdes au niveau du générateur hypothalamique de GnRH est actuellement considérée comme la cause majeure des aménorrhées dites "psychogènes " (anorexie mentale, aménorrhée des sportives, etc.) $[10,11,13,17]$ en raison de l'action de rétroaction négative des opioïdes sur la pulsatilité de la GnRH [6, 8, 20], il semble vraisemblable que les opioïdes, voire d'autres neurohormones, puissent altérer sans l'effondrer la pulsatilité de GnRH entraînant secondairement une dysovulation. Ainsi, pour certains auteurs [11, 13], une hypersécrétion de CRH (corticotropin releasing hormone) serait à l'origine des anomalies de la pulsatilité de $\mathrm{GnRH}$ responsable de troubles de l'ovulation [13]. Chez les femmes hypersportives chez lesquelles on ne note cependant pas d'aménorrhée, on observe une diminution de la fréquence des pulsations de $\mathrm{GnRH}$ assortie d'une exagération de l'amplitude de celles-ci [13].

On trouve dans cet exemple la démonstration selon laquelle des anomalies de la pulsatilité de GnRH sont susceptibles d'engendrer une simple insuffisance lutéale en rapport direct avec une anomalie du générateur hypothalamique [4]. Ces constatations accréditent, en outre, les observations dé jà anciennes de Matsumoto et al. [14] montrant que les agressions psychologiques de la vie quotidienne pourraient provoquer des altérations de la courbe ménothermique des femmes.

La preuve a contrario du rôle des neurohormones dans l'altération de la pulsatilité de $\mathrm{GnRH}$ a été fournie par certains auteurs qui ont montré que l'administration d'antagonistes des opiacés (naltrexone) à des femmes souffrant d'anorexie mentale était capable de provoquer un rétablissement des cycles ovulatoires [21].

On peut donc conclure que certaines dysovulations peuvent être causées par des anomalies de la pulsatilité de $\mathrm{GnRH}$ relevant de causes soit structurelles soit acquises, notamment à l'occasion d'agressions psychogènes ou vécues comme telles (condition de travail mal adaptées, environnement stressant, sport intensif, notamment de compétition) [1].

Qu'en est-il des causes exogènes? Tout d'abord, précisons que nous restreignons ce terme aux médicaments utilisés en reproduction et plus spécialement aux estroprogestatifs à visée contraceptive et aux progestatifs utilisés largement en France, soit pour pallier des symptômes en rapport avec une insuffisance lutéale (vraie ou présumée), soit comme contraceptifs, notamment chez les femmes pour lesquelles la contraception estroprogestative est contre-indiquée en raison de risques métaboliques ou vasculaires (thrombo-embolie, maladie de système, diabète, hypertension artérielle, etc.).

En ce qui concerne les estroprogestatifs, on a assisté depuis vingt ans à la réduction des doses d'estrogènes et de progestatifs au sein des pilules contraceptives dans le but louable de diminuer voire d'annuler leur effet hépatique toxique, essentiellement la synthèse de certaines lipoprotéines, de facteurs de coagulation, du substrat rénine, etc. Ainsi a-t-on vu s'abaisser le contenu d'éthinyl-estradiol de $100 \mu \mathrm{g}$ par pilule à successivement 50 puis 30 voire $20 \mu \mathrm{g}$. A ces deux dernières doses, l'effet de rétroaction négative que l'éthinyl-estradiol est censé exercer sur l'hypothalamus médian n'est pas assuré. En revanche, l'effet de rétroaction positive hypophysaire entraînant la libération de FSH et de LH persiste vraisemblablement. On manque totalement d'informations sur l'action de 20 ou $30 \mu \mathrm{g}$ d'éthinyl-estradiol sur la pulsatilité de la GnRH appréciée à travers les "pulses" de LH chez la femme normale soumise à ce schéma thérapeutique.

Concernant les progestatifs associés à l'éthinyl-estradiol, on a abandonné progressivement les norstéroïdes, considérés à juste titre comme constituant un risque métabolique et vasculaire potentiel, au profit de dérivés prégnane ou norprégnane, voire de molécules plus récentes (désogestrel, norgestimate et gestodène). Ces progestatifs ont-ils une activité antigonadotrope suffisante? Des dosages ponctuels d'estradiol plasmatique pratiqués chez des femmes utilisant ces associations (30 à $40 \mu \mathrm{g}$ d'éthinylestradiol + un progestatif dit de $3^{e}$ génération en association continue ou en palier) ne montrent que rarement des chiffres élevés. Toutefois, l'effet antigonadotrope de ces nouvelles associations ne peut pas être affirmé sur ces seules données. S'il semble évident que le pic ovulatoire de LH est supprimé lors de leur administration $[2,3]$, aucun document ne relate leur action sur la pulsatilité de LH. Autrement dit, rien ne permet d'affirmer que ces molécules ne sont pas génératrices d'anomalies au niveau de la fréquence et de l'amplitude de $\mathrm{GnRH}$ pouvant conduire, si la pulsatilité de $\mathrm{GnRH}$ n'est pas abolie, à une dysovulation comme nous l'avons évoqué plus haut.

En ce qui concerne la thérapeutique progestative à base de dérivés prégnane et norprégnane administrés chez la femme du $5^{e}$ jour - voire du $10^{e}-$ au $25^{e}$ jour du cycle, l'incertitude quant à un effet antigonadotrope est non moins grande. Si ces composés ont un effet antiestrogène et, surtout, progestogène évident au niveau de l'endomètre, leur action au niveau de la glande mammaire est beaucoup moins nette. En témoigne la difficulté à réduire les manif estations mammaires d'hyperestrogénie (mastodynies, etc.) avec ces composés utilisés même à forte dose. Cet effet paradoxal contraste avec l'efficacité des norstéroïdes fortement antigonadotropes (Noréthistérone, Lynestrénol, etc.) voire avec l'activité antiestrogène in vitro de ces composés. Ainsi la Surgestone (Promégestone), très active in vitro comme suppresseur de l'activité mitotique de l'estradiol sur les cellules épithéliales mammaires normales en culture [9], a souvent très peu d'effet in vivo sur les affections mammaires bénignes. Là encore, il est permis d'évoquer un effet insuffisant de ce composé en terme d'abolition de la pulsatilité de la GnRH.

\section{En conclusion}

La chronobiologie hormonale a permis, en moins de dix ans, de redéfinir la physiologie du cycle menstruel en clarifiant l'effet de la GnRH sur sa réalisation ainsi que le rôle des différentes rétroactions positives et négatives exercées par les stéroïdes sexuels et les neurotransmetteurs présents 
dans l'hypothalamus. Cela, aussi bien dans les conditions normales que dans certaines situations pathologiques.

Une nouvelle classification des désordres ovulatoires constitutionnels ou provoqués est en cours d'établissement. Ainsi, l'aménorrhée de l'anorexie mentale et de diverses origines psychogènes, voire environnementales, s'éclaire d'un jour nouveau permettant d'entrevoir des approches thérapeutiques mieux adaptées. Le concept vague de dysovulation luimême se précise à la lumière de la chronobiologie, lorsque celle-ci fait appel à des critères rigoureux d'étude et d'évaluation. L'action des médicaments largement utilisés en gynécologie, notamment les contraceptifs hormonaux et les progestatifs, est à notre sens appréciée avec des critères désormais obsolètes. Il importe de faire la preuve non seulement de leur efficacité, mais encore de leur mode d'action, de leur iatrogénicité éventuelle avec les méthodes utilisées chez les primates puis chez la femme en physiologie.

L'endocrinologie de la reproduction doit donc faire l'effort de s'adapter en faisant appel à la chronobiologie moderne, si elle ne veut pas rester empirique, ce qui est hélas trop souvent le cas actuellement

\section{P. Mauvais-Jarvis}

Professeur, chef du service d'endocrinologie et de médecine de la reproduction, hôpital Necker, 149 rue de Sèures, 75743 Paris Cedex 15, France.

\section{RÉFÉRENCES}

1. Armstrong DT. Environmental stress and ovarian function. Biology of Reproduction 1986 ; 34 : 29-39.

2. Bazin B, Thévenot $\mathrm{R}$, Bursaux $\mathrm{C}$, Paris J. Effect of Nomegestrol acetate, a new 19 nor-progesterone derivative, on pituitary ovarian function in women. Brit J Obst Gynec 1987; 94 : 1199-204.

3. Blacker C, Senet JM, Dubourg D, Denis C, Fourtillan JB. Action de la promégestone à la dose de $0,500 \mathrm{mg} / \mathrm{j}$ vingt jours par mois sur le pic ovulatoire de $\mathrm{LH}$. Horm Repr Metab 1987 ; 3 : 283-8.

4. Crowley WF, Filicori M, Sprattd I, Santoro NF. The physiology of gonadotrophin releasing hormone ( $\mathrm{GnRH})$ secretion in men and women. Recent Progr Horm Res 1985 ; 41 : 473-526.

5. Cumming DC, Vickovic MM, Wall SR, Fluker MR. Defects in pulsatile LH release in normally menstruating runners. $J$ Clin Endocrinol Metab $1985 ; 60$ : 810-2.

6. Ferin M, Van Vugt D, Wardlaw S. The hypothalamic control of the menstrual cycle and the role of endogenous opioid peptides. Recent Prog Horm Res 1984 ; 40 : 441-85.

7. Gindoff PR, Ferin M. Endogenous opioid peptides modulate the effect of corticotropin-releasing factor on gonadotropin release in the primate. Endocrinology $1987 ; 121$ : 837-42.

8. Gindoff PR, Jewelewicz R, Membree W, Wardlaw S, Ferin M. Sustained effects of opioïd antagonism during the normal human luteal phase. $J$ Clin Endocrinol Metal $1988 ; 66: 1000-4$

9. Gompel A, Malet C, Spritzer P, Lalardrie JP, Kuttenn F, Mauvais-Jarvis P. Progestin effect on cell proliferation and $17 \beta$ hydroxysteroid dehydrogenase activity in normal breast cells in culture. $J$ Clin Endocrinol Metab 1986 ; 63 : 1174-80.

10. Hotta M, Shibasaki T, Masuda A, et al. The responses of plasma adrenocorticotropin and cortisol to corticotropin-releasing hormone (CRH) and cerebrospinal fluid immunoreactive $\mathrm{CRH}$ in anorexia nervosa patients. J Clin Endocrinol Metab 1986 ; 62 : 319-24.

11. Kaye WH, Gwirtsman HE, George DT, et al. Elevated cerebrospinal fluid levels of immunoreactive corticotropinreleasing hormone in anorexia nervosa relation to state of nutrition, adrenal function, and intensity of depression. J Clin Endocrinol Metab 1987 ; 64 : 203-8.

12. Knobil E. The neuroendocrine control of the menstrual cycle. Recent Prog Horm Res $1980 ; 36$ : 53-88.

13. Looks AB, Mortola JF, Girton L, Yen SSC. Alterations in the hypothalamic, pituitary, ovarian and the hypothalamic, pituitary, adrenal axis in atletic women. $J$ Clin Endocrinol Metab 1989 ; 68 : 402.

14. Matsumoto $\mathrm{S}$, Igarashi $\mathrm{M}$, Wagauba Y. Environmental anovulatory cycles. Int J Fert 1968 ; 13 : 15-23.

15. Mauvais-Jarvis P, Bricaire C. Pathophysiology of polycystic ovary syndrome. $J$ Steroid Biochem 1989 ; 33 : 791-4.

16. Mauvais-Jarvis P, Kuttenn F. Les anovulations et les dysovulations. In : Encyclopedie Med Chir. 1975; Endocrinologie $10027 \mathrm{C}^{10}$

17. Quigley ME, Sheehan KI, Casper RF, Yen SSC. Evidence for increased dopaminergic and opioid activity in hypothalamic hypogonadotropic amenorrhea. J Clin Endocrinol Metab. 1980 ; 50 : 949-53.

18. Rebar R, Judd HC, Yen SSC. Characterization of the inappropriate gonadotropic secretion polycystic ovarian syndrome. J Clin Invest $1976 ; 57$ : 1320-9. 19. Sherman BM, Korenman SG. Hormonal characteristics of the human menstrual cycle throughout reproductive life. J Clin Invest $1975 ; 55$ : 699-706.

20. Wehrenberg WB, Wardlaw SL, Frantz AG, Ferin M. $\beta$-endorphin in hypophyseal portal blood: variations throught the menstrual cycle. Endocrinology 1982 ; $111: 879-81$

21. Wildt L, Leyendecker G. Induction of ovulation by the chronic administration of naltrexone in hypothalamic amenorrhea. $J$ Clin Endocrinol Metab 1987 ; 64 : 1334-5.

\section{Summary}

The dysfunction of the female GnRH pulse generator revisited

The systemic pulsatile nature of gonadotropin secretion first noted in the ovariectomized rhesus monkey has since been described in other mammals including the women.

So, a normal menstrual cycle is the result of complexe interactions between the hypothalamus which secrete gonadotropin releasing hormone $(\mathrm{GnRH})$ on a pulsatile mode, the pituitary with secretion $\mathrm{LH}$ and FSH and the ovaries whose two main products of secretion are estradiol and progesterone. In addition, a group of substances named neurohormones, particularly the endogenous opioid peptides may exert a pivotal role in the control of the menstrual cycle. Recently, abnormalities in $\mathrm{GnRH}$ pulsatility has been observed in psychogenic amenorrheas such as anorexia nervosa and also in athletic training. Abnormal frequency and amplitude of $\mathrm{GnRH}$ pulses have also been observed in polycystic ovarian syndrome (PCO) and in various aspects of luteal phase insufficiency very close to PCO.

Prospectively, it would be interesting to know how contraceptive steroids containing very low doses of estrogens and progestins are acting on GnRH pulsatility in order to be sure that these compounds are able to completely suppress the $\mathrm{GnRH}$ secretion or not. In the latter case they could be responsible of $\mathrm{GnRH}$ pulse generator dysregulation and further of ovarian dysfunction. This is also the case of various synthetic progestins newly synthesized and used in France.

In other words, in addition to clinical situations which are now currently evaluated by way of LH pulsatility studies, the antigonadotropic actions of drugs used in contraception or in endocrinological gynaecology might be approached by the same experimental procedure in order to know more precisely their mode of action and their safety in normal women. 